\title{
Assessing the new ICD-MM classification for assigning the cause of maternal mortality at a tertiary centre in Western India: a retrospective study
}

\author{
Tosha M. Sheth*, Palak P. Vaishnav, Nandita K. Maitra
}

Department of Obstetrics and Gynecology, Medical College Baroda and Sir Sayajirao General Hospital, Vadodara, Gujarat, India

Received: 11 June 2019

Accepted: 16 July 2019

*Correspondence:

Dr. Tosha M. Sheth,

E-mail: drtoshasheth@gmail.com

Copyright: (c) the author(s), publisher and licensee Medip Academy. This is an open-access article distributed under the terms of the Creative Commons Attribution Non-Commercial License, which permits unrestricted non-commercial use, distxribution, and reproduction in any medium, provided the original work is properly cited.

\begin{abstract}
Background: The World Health Organisation (WHO) in 2012 introduced the $10^{\text {th }}$ revision of International Classification of Disease (ICD 10) to deaths in pregnancy, labour and puerperium (ICD-MM) for consistent collection, analysis and interpretation of information on maternal deaths. The proper use of this classification requires training to avoid heterogeneity and error in the classification of maternal deaths.

Methods: We analysed the Maternal Death Review (MDR) forms of 295 deaths over a period of 5 years (January 2014 to December 2018 inclusive) occurring at a tertiary health centre in Western India. The ICD-MM classification was used to reassign the cause of death.

Results: There were 295 deaths in women during pregnancy, childbirth and puerperium during the 5 year period. Of these there were 294 maternal deaths and one coincidental death. There were 173 deaths of the direct type (58.84\%), 105 deaths of the indirect type (35.71\%) and 16 deaths $(5.44 \%)$ of the unspecified type. Obstetric haemorrhage was the highest contributor to direct deaths $(23.8 \%)$ and anaemia contributed to the maximum deaths from indirect causes $(13.6 \%)$ followed by liver diseases in pregnancy $(10.54 \%)$.Unanticipated complications of management accounted for $2 \%$ of the total deaths. There was considerable inaccuracy in assigning cause of death by consultants who were untrained in the use of the ICD-MM classification.

Conclusions: ICD-MM classification promotes an accurate assignment of the cause of death. Training of healthcare providers performing maternal death reviews in the use of this classification is essential to identify accurate underlying cause of death and contributory conditions.
\end{abstract}

Keywords: Contributory conditions, ICD-MM, Maternal mortality, Maternal death review, Underlying cause of death

\section{INTRODUCTION}

In the present day, ending preventable maternal mortality (EPMM) is one of the most challenging problems and an unaccomplished target in most countries. Since 1990, there has been a decrease in maternal deaths worldwide by $45 \%$ but, 800 women still die each day from largely preventable causes before, during, and after giving birth.
Millennium Development Goals require all countries to reduce their Maternal Mortality Ratio (MMR) by at least two thirds of their 2010 baseline level by 2030. The average global target is an MMR of less than 70/100 000 live births by $2030 .^{1}$

Poor understanding of the root cause of death and assigning multiple contributing conditions as the primary 
cause of death are some of the major challenges that limit effective implementation of preventive and control strategies of maternal mortality.

As the countries move towards development and reduced maternal mortality rates, there is a shift in cause of death from direct causes to indirect causes; a phenomenon called 'obstetric transition'.2

An accurate classification of the cause of maternal death is vital in order to plan targeted interventions to achieve the goal of ending preventable maternal mortality.

The application of the $10^{\text {th }}$ revision of International Classification of Disease (ICD 10) to deaths in pregnancy, labour and puerperium (ICD-MM) was introduced by the World Health Organisation (WHO) in 2012 with the aim of facilitating consistent collection, analysis and interpretation of information on maternal deaths worldwide. ${ }^{3}$

The aim of this study was to analyse the facility based Maternal Death Review (MDR) records over a period of 5 years and to study the implication of reclassification of the cause of death according to the ICD-MM classification.

\section{METHODS}

In the present study, all deaths in women during pregnancy, childbirth and puerperium that occurred in a tertiary care centre, Baroda Medical College, Gujarat, Western India during a 5 year period (January 2014 to December 2018 inclusive) were studied. This is a referral centre with patients referred from around 42 Primary Health Centres, 10 Community Health Centres, 34 Urban Primary Health Centres, one district hospital, neighbouring districts and two neighbouring states of Madhya Pradesh and Rajasthan.

\section{Inclusion criteria}

were all deaths occurring during pregnancy, childbirth and puerperium up to 42 days from obstetric complications of the pregnancy state (direct obstetric deaths), from previous existing disease or disease that developed during pregnancy which was aggravated by the physiological effects of pregnancy (indirect obstetric deaths), deaths resulting from interventions, omissions or incorrect treatment or those in which the cause was unknown during pregnancy, childbirth or puerperium up to 42 days.

\section{Exclusion criteria}

All deaths occurring from accidental or incidental causes during pregnancy, childbirth or puerperium were excluded from the study and the analysis.
Facility based Maternal Death Review (MDR) forms were filled for every maternal death occurring not only in the maternity wards and labour room but, also in any section of the health facility. The cause of death was assigned by the consultant who was in charge of the care of the patient. Not all consultants were trained in ICDMM system of classifying maternal deaths. This resulted in heterogeneity in the assignment of the cause of death.

The MDR forms of all maternal deaths over a 5 year period were studied. The case summary was reviewed after which the ICD-MM classification was used to reassign the cause of death. The underlying cause of death is defined by the ICD-MM as "the condition or disease that initiated the morbid chain of events leading to death". Contributing conditions are defined as "conditions present that may have contributed to (or be associated with) but did not directly cause death". ${ }^{3}$ There could be only one underlying cause of death but multiple contributing conditions leading to death in a given patient. In the ICD-MM classification, three types of maternal deaths are defined: direct, indirect and unspecified. There are eight different groups of underlying causes and the ninth group is used for coincidental causes not related to or aggravated by pregnancy or its management. ${ }^{3}$

\section{RESULTS}

There were 295 mortalities in women during pregnancy, childbirth and puerperium over the 5 year study period. Analysis of the maternal death review (MDR) forms revealed that there was no standardisation of the terminologies used to assign maternal death. The underlying cause was referred to either as 'primary cause', 'likely cause' or 'final cause' leading to confusion. There was no clarity on what the term 'secondary cause' of death referred to. It probably referred to the contributing conditions associated with maternal death.

The other important fallacy that was noted was that there were multiple conditions which were designated as the underlying or primary cause of death. The ICD-MM states that there can be only one underlying cause of death which initiated the train of morbid events leading directly to death.

In spite of the variation in the terminologies used to describe the underlying cause of death, it was possible to reassign the cause of death and designate a type and group according to the ICD-MM classification to 279 out of 295 cases based on review of the case summary.

According to the ICD-MM classification, out of the 295 deaths in women during pregnancy, childbirth and puerperium during the study period, 294 were classified as maternal deaths and one case was classified as coincidental death. 
Of the 294 maternal deaths, there were 173 direct deaths, 105 indirect deaths and 16 cases where the cause of death was unknown or unspecified (Table 1).

\section{Reclassification into groups}

For almost all cases, a group could be identified according to the ICD-MM classification (Table 1).

Table 1: ICD-MM Classification.

\begin{tabular}{|c|c|c|c|}
\hline \multirow{2}{*}{\multicolumn{4}{|c|}{$\begin{array}{l}\text { ICD-MIM type ICD-MM group } \\
\text { A. maternal death }(n=294)\end{array}$}} \\
\hline & & & \\
\hline \multirow[t]{7}{*}{ Direct maternal death } & & & $173(58.84)$ \\
\hline & 1 & Pregnancy with abortive outcome & $7(2.38)$ \\
\hline & 2 & $\begin{array}{l}\text { Hypertensive disorder of pregnancy, child birth and } \\
\text { peurperium }\end{array}$ & $55(18.70)$ \\
\hline & 3 & Obstetric haemorrhage & $71(23.80)$ \\
\hline & 4 & Pregnancy related infections & $20(6.80)$ \\
\hline & 5 & Other obstetric complications & $14(4.76)$ \\
\hline & 6 & Unanticipated complications of management & $6(2.04)$ \\
\hline \multirow{8}{*}{ Indirect maternal death } & 7 & Non-obstetric complications & $105(35.71)$ \\
\hline & & Anaemia & $40(13.60)$ \\
\hline & & Cardiac disorders & $9(3.06)$ \\
\hline & & Liver disorders & $31(10.54)$ \\
\hline & & Respiratory disorders & $6(2.04)$ \\
\hline & & Neurological disorders & $3(1.02)$ \\
\hline & & Infections/infestations & $15(5.10)$ \\
\hline & & Neoplasm & $1(0.34)$ \\
\hline Unspecified & 8 & Unknown/undetermined cause & $16(5.44)$ \\
\hline
\end{tabular}

\section{Cases for group 'obstetric haemorrhage'}

Obstetric haemorrhage accounted for $23.8 \%$ of cases of maternal deaths and was the leading cause of direct maternal deaths accounting 71 out of 173 cases (41.04\%) of all direct maternal deaths (Figure 1).

\section{Cases for group 'unanticipated complications of management"}

There were 6 cases which were reassigned to this group. In this group there were 3 cases where death was directly attributable to anaesthetic complications. In the previous classifications there was no group to which such deaths could be assigned leading to such deaths missing from the calculated statistics for direct maternal deaths.

Similarly, there was one death due to Transfusion related Acute Lung Injury (TRALI) and one death due to blood transfusion reaction which are important complications of management which would previously go unreported and have now been included in this group. There was one case of death following penicillin injection in a patient with Rheumatic Heart Disease which could be assigned this group.

\section{Cases for type 'indirect deaths'}

There were 105 cases of indirect maternal deaths accounting for $35.71 \%$ of all maternal deaths. This was thus the largest group contributing to maternal deaths. Among all the causes of indirect maternal deaths, anaemia was the most prevalent accounting for 40 out of the 105 cases $(38.09 \%)$ of indirect maternal deaths in the study period. Among patients in whom anaemia was identified as the indirect cause of death, 10 cases $(25 \%)$ were due to iron deficiency anaemia, 27 cases $(67.5 \%)$ were due to sickle cell anaemia and 3 cases $(7.5 \%)$ were due to aplastic anaemia. The high prevalence of sickle cell anaemia in this geographic area explains why this condition was responsible for $67.5 \%$ of the indirect deaths due to anaemia in our study population. ${ }^{4}$

Liver diseases contributed to the second largest number of cases of indirect maternal deaths accounting for 31 out of $105(29.52 \%)$ indirect cases of death. $32.26 \%$ of these deaths were secondary to Hepatitis $\mathrm{E}$ viral infection, $16.12 \%$ were secondary to acute hepatic failure with sickling positivity and $45.16 \%$ were secondary to acute hepatic failure with sickling negativity (Figure 2). 


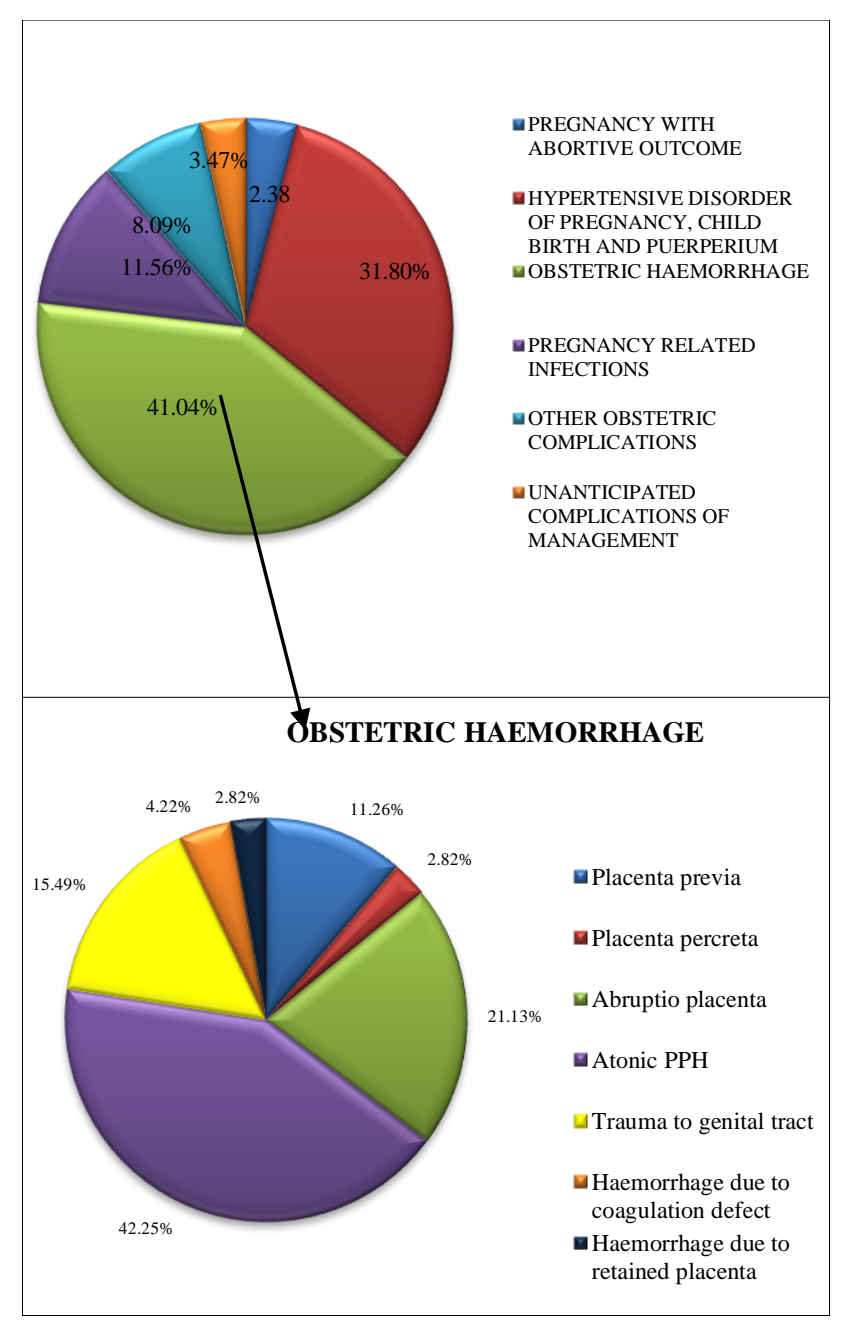

Figure 1: Direct causes of maternal death.

Using the ICD-MM classification it was possible to designate a specific underlying cause to each of the 71 deaths occurring in this group. Among the cases of antepartum haemorrhage, there were 8 cases of placenta previa (11.26\%), 2 cases of placenta percreta $(2.82 \%)$ and 15 cases of abruptio placenta $(21.13 \%)$. Among cases with postpartum haemorrhage it was possible to identify that 30 cases were due to atonic PPH (42.25\%), 11 cases were secondary to genital tract trauma $(15.49 \%)$. There were 2 cases due to retained placenta $(2.82 \%)$ and 3 cases secondary to haemorrhage due to disorders of blood coagulation $(4.22 \%)$. A classification in this format using the ICD-MM classification would provide a useful insight into the specific underlying cause of death for implementation of effective preventive measures (Figure $1)$.

\section{Contributing conditions}

Anaemia was the leading contributory condition associated with maternal deaths in the study group. Hypertensive disorders of pregnancy were the second most common contributory condition.

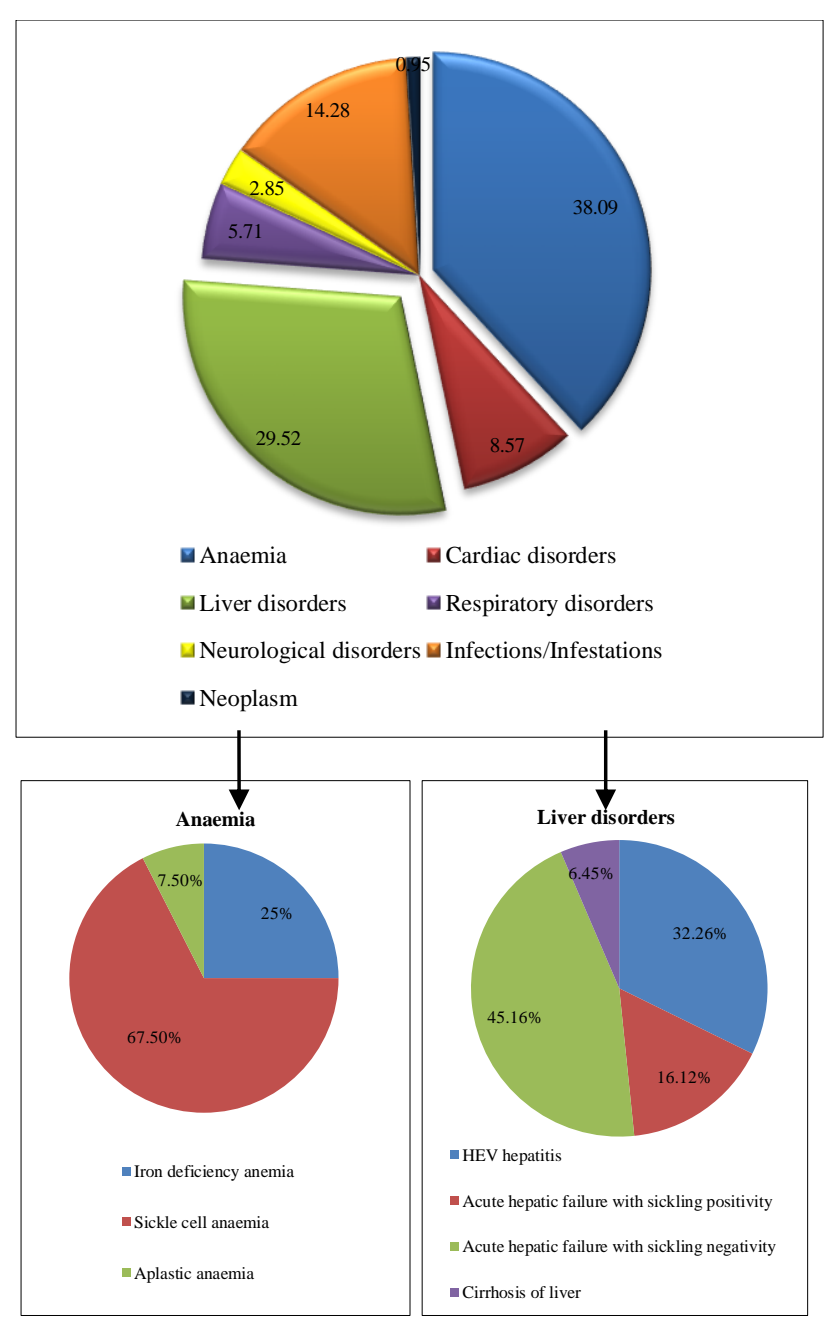

Figure 2: Indirect causes of maternal deaths.

\section{DISCUSSION}

In this study, we analyzed the facility based Maternal Death Review (MDR) records of all deaths over a 5 year period and reassigned the cause of death based on the ICD-MM classification. We studied the implication of the reclassification on the implementation of preventive and control strategies to reduce maternal deaths in our population.

We found that there were marked fallacies in the assignment of the cause of death by the consultants most of whom were untrained in the use of the ICD-MM classification. There was no clarity on the concept of the 'underlying cause of death and the 'contributory conditions'. Multiple causes were designated as the 'primary cause of death' instead of only one underlying cause.

This study demonstrates that it is possible by reviewing the case summary to clearly identify an underlying cause of death in most cases with a clearly designated type and group using the ICD-MM classification. 
A review of the facility based Maternal Death Review forms and the reassignment of the cause of death according to clearly defined groups brought clarity to the exact underlying cause of death in our study population.

Indirect causes contributed to the highest number of mortalities which is in line with the principle of 'obstetric transition' which states that as the rates of maternal mortality decrease, there is a shift from direct to indirect causes of death. Sickle cell anaemia is widely prevalent in the geographical area where this study was conducted and the huge burden of cases attributed to this condition alone would highlight the need to focus efforts on identification of patients with this condition in the antenatal period and to institute measures to correct anaemia before the patient goes into labour and avoidance of factors that would precipitate sickle cell crisis in these patients.

The other significant finding of this study was that in spite of all measures employed to reduce deaths due to obstetric haemorrhage, it is still the leading direct cause of maternal deaths in this part of the world.

Liver diseases in pregnancy emerged as an important indirect cause of death in our population and the aetiology of acute liver failure remained undetermined in a large majority of these patients.

Without a clear identification of the cause of death, it is impossible to focus resources on areas which require maximum attention to progress towards the goal of ending preventable maternal mortality. The cause of deaths are highly variable in different parts of the world and thus a study of this kind in our population was required to identify the conditions which contributed to the highest number of maternal deaths in our area and redirect resources, manpower and control measures specifically towards these conditions.

The findings of this study are in agreement with a similar study carried out in Sri Lanka by Agampodi et al in which the maternal deaths were reclassified according to the ICD- MM classification. This lead to identification of causes which were highly prevalent in their population and were brought to light due to reclassification. ${ }^{5}$ Another similar study was performed in the United Kingdom by Mgawadere et al who compared the assignment of maternal death by a facility based review team, an expert panel using ICD-MM and a computer based program and concluded that training in the use of ICD-MM is required in order to correctly assign the cause of death. ${ }^{6}$

There was poor agreement between the cause of death provided by healthcare providers and an expert panel using ICD-MM in a study conducted in Malawi. ${ }^{7}$ Inaccurate reporting of the cause of death would limit the usefulness of the data on maternal mortality for the purpose of framing a policy, resource allocation and mobilization of other public health measures. ${ }^{8,9}$
This study also highlighted the need to make the Maternal Death Review forms easy to understand, simple to fill and standardized. Only then there would be a good documentation of maternal deaths. The use of the ICDMM classification would make the classification of deaths uniform, easy to assign by the health care professionals and easy to comprehend for the policy makers.

The policy makers focus attention primarily on well researched direct causes of death like obstetric haemorrhage and hypertensive disorders in pregnancy. Condition such as anaemia is an important indirect cause of death as well as a significant contributing factor in much maternal mortality but is often overlooked. The use of ICD-MM draws attention to all such causes of death which are otherwise ignored.

\section{CONCLUSION}

The ICD-MM classification is the standard format in which maternal deaths should be assigned and reported worldwide. It results in uniformity in the classification of maternal deaths. This would draw attention of health planners to focus attention on implementation of programs and allocation of resources specific to the causes of death most prevalent in the concerned population and help us move closer to our goal of ending preventable maternal mortality. Training of health care providers performing maternal death reviews in the use of this classification is the first step in this direction.

Funding: No funding sources

Conflict of interest: None declared

Ethical approval: Not required

\section{REFERENCES}

1. World Health Organisation. Strategies towards ending preventable maternal mortality (EPMM). Geneva: World Health Organisation, 2015. Available at:

who.int/reproductivehealth/topics/maternal_perinatal /epmm/en/. Accessed on 28 January 2019.

2. Souza JP, Tunçalp Ö, Vogel JP, Bohren M, Widmer M, Oladapo OT, et al. Obstetric transition: the pathway towards ending preventable maternal deaths. BJOG. 2014;121 Suppl.1:1-4.

3. World Health Organization. The WHO Application of ICD-10 to Deaths During Pregnancy, Childbirth and the Puerperium: ICD-MM. Geneva: World Health Organization, 2012. Available at: www.who.int/reproductivehealth/publications/monit oring/9789241548458/en/. Accessed on 28 January 2019.

4. Patel AP, Naik MR, Shah NM, Sharma N, Parmar P. Prevalence of common hemoglobinopathies in Gujarat: an analysis of a large population screening programme. Natl J Community Med. 2012;3:112-6. 
5. Agampodi S, Wickramage K, Agampodi T, Thennakoon U, Jayathilaka N, Karunarathna D, et al. Maternal mortality revisited: the application of the new ICD-MM classification system in reference to maternal deaths in Sri Lanka. Reprod Health. 2014;11(1): 17.

6. Mgawadere F, Unkels $\mathrm{R}$, van den Broek $\mathrm{N}$. Assigning cause of maternal death: a comparison of findings by a facility-based review team, an expert panel using the new ICD-MM cause classification and a computer-based program (interval-4). BJOG. 2016;123:1647-53.

7. Owolabi H, Ameh C, Bar-Zeev S, Adaji S, Kachale F, van den Broek N. Establishing cause of maternal death in Malawi via facility-based review and application of ICD-MM classification. BJOG. 2014;121:95-101.
8. Kircher T, Anderson RE. Cause of death. Proper completion of the death certificate. J Am Med Assoc. 1987;258:349-52.

9. Messite J, Stellman SD. Accuracy of death certificate completion: the need for formalized physician training. J Am Med Assoc. 1996;275:7946.

Cite this article as: Sheth TM, Vaishnav PP, Maitra NK. Assessing the new ICD-MM classification for assigning the cause of maternal mortality at a tertiary centre in Western India: a retrospective study. Int J Reprod Contracept Obstet Gynecol 2019;8:3629-34. 